\title{
THE POLAR CATALYSMIC VARIABLE 1RXS J173006.4+033813
}

\author{
Varun B. Bhalerao ${ }^{1}$, Marten H. van KerkwiJK ${ }^{1,3}$, Fiona A. Harrison ${ }^{2}$, \\ Mansi M. Kasliwal ${ }^{1}$, S. R. KulKarni ${ }^{1}$, And Vikram R. Rana ${ }^{2}$ \\ ${ }^{1}$ Department of Astronomy, California Institute of Technology, Pasadena, CA 91125, USA \\ ${ }^{2}$ Space Radiation Laboratory, California Institute of Technology, Pasadena, CA 91125, USA \\ Received 2010 January 27; accepted 2010 July 27; published 2010 August 27
}

\begin{abstract}
We report the discovery of 1RXS J173006.4+033813, a polar cataclysmic variable with a period of 120.21 minutes. The white dwarf primary has a magnetic field of $B=42_{-5}^{+6} \mathrm{MG}$ and the secondary is an M3 dwarf. The system shows highly symmetric double-peaked photometric modulation in the active state as well as in quiescence. These arise from a combination of cyclotron beaming and ellipsoidal modulation. The projected orbital velocity of the secondary is $K_{2}=390 \pm 4 \mathrm{~km} \mathrm{~s}^{-1}$. We place an upper limit of $830 \pm 65 \mathrm{pc}$ on the distance.
\end{abstract}

Key words: binaries: close - binaries: spectroscopic - novae, cataclysmic variables - stars: individual (1RXS J173006.4+033813) - stars: variables: general

Online-only material: color figures, machine-readable tables

\section{INTRODUCTION}

Cataclysmic variables (CVs) are close interacting binary systems in which a white dwarf (WD) accretes material from a Roche lobe-filling late-type secondary star (Warner 1995; Hellier 2001). In most non-magnetic CVs $\left(B<10^{4} \mathrm{G}\right)$, the material lost from the secondary does not directly fall onto the WD because of its large specific orbital momentum: instead, it settles down in an accretion disk around the WD. The accretion disk is the brightest component of the $\mathrm{CV}$ due to the large gravitational energy release in viscous accretion. The disk dominates the emission from the WD and donor over a wide wavelength range.

On the other hand, the accretion geometry in magnetic CVs is strongly influenced by the WD magnetic field. Magnetic CVs are broadly divided into two subclasses: polars and Intermediate Polars (IPs). Polars usually show a synchronous or near synchronous rotation of a WD with the orbital motion of the binary system and have high magnetic fields $(B>10 \mathrm{MG}$ ) (for a review, see Cropper 1990). In IPs the WD rotation is far from synchronous and typically has a magnetic field, $B<10 \mathrm{MG}$ (for a review, see Hellier 2002). The strong magnetic field in polars deflects the accretion material from a ballistic trajectory before an accretion disk can form, channeling it to the WD magnetic pole(s). The infalling material forms a shock near the WD surface, which produces radiation from X-rays to infrared wavelengths. Electrons in the ionized shocked region spiral around the magnetic field lines and emit strongly polarized cyclotron radiation at optical and infrared wavelengths. Polars exhibit $\mathrm{X}$-ray on (high) and off (low) states more frequently than the other variety of CVs (Ramsay et al. 2004).

1RXS J173006.4+033813 (hereafter 1RXS J1730+03) is a Galactic source that is highly variable in the optical and X-ray, exhibiting dramatic outbursts of more than 3 mag in the optical. It was discovered by the ROSAT satellite during its all-sky survey (Voges et al. 1999). Denisenko et al. (2009), in the course of their investigation of poorly studied ROSAT sources, reported that USNO-B 1.0 object 0936-00303814, which is within the $10^{\prime \prime}$ (radius) localization of the X-ray source showed great variability ( $\Delta R$ of up to $3 \mathrm{mag}$ ) in archival data (Palomar Sky Survey;

\footnotetext{
3 On sabbatical leave from the Department of Astronomy and Astrophysics, University of Toronto, 50 St. George Street, Toronto, ON M5S 3H4, Canada.
}

SkyMorph/NEAT). During certain epochs, the source appears to have been undetectable ( $\left.m_{R}>20 \mathrm{mag}\right)$. Denisenko et al. (2009) undertook observations with Kazan State University's $30 \mathrm{~cm}$ robotic telescope and found variability on rapid timescales of 10 minutes.

In this paper, we report the results of our photometric, spectroscopic, and X-ray follow-up of 1RXS J1730+03.

\section{OBSERVATIONS}

\subsection{Optical Photometry}

We observed 1RXS J1730+03 with the Palomar Robotic 60 inch telescope (P60; Cenko et al. 2006) from UT 2009 April 17 to UT 2009 June 5 and with the Large Format Camera (LFC; Simcoe et al. 2000) at the $5 \mathrm{~m}$ Hale telescope at Palomar on UT 2009 August 26. Here, we give details of the photometry.

We define a photometric epoch as observations from a single night when the source could be observed. We obtained 28 epochs with the P60, subject to scheduling and weather constraints. A typical epoch consists of consecutive 90-120 s exposures spanning between 30 and 300 minutes (Table 1). We obtained $g^{\prime}, r^{\prime}, i^{\prime}$ photometry on the first and third epochs. After the third epoch, we continued monitoring the source only in the $i^{\prime}$ band.

We reduced the raw images using the default P60 image analysis pipeline. LFC images were reduced in IRAF. ${ }^{4} \mathrm{We}$ performed photometry using the IDL $^{5}$ DAOPHOT package (Landsman 1993). Fluxes of the target and reference stars (Figure 1) were extracted using the APER routine. For aperture photometry, the extraction region was set to one seeing radius, as recommended by Mighell (1999). The sky background was extracted from an annular region 5-15 seeing radii wide. We used flux zero points and seeing values output by the P60 analysis pipeline. Magnitudes for the reference stars were calculated from a few images (Table 2). The magnitude of 1RXS $\mathrm{J} 1730+03$ was calculated relative to the mean magnitude of 9 reference stars for LFC images and 15 reference stars for P60 images. The LFC images (Figure 1) resolve out a faint nearby star $\left(m_{i^{\prime}}=20.8\right), 3^{\prime \prime}$. 4 from the target. The median seeing in P60 data is 2". 1 (Gaussian FWHM): so there is a slight contribution from the flux of this star to photometry of 1RXS J1730+03. We

\footnotetext{
4 http://iraf.noao.edu/

5 http://www.ittvis.com/ProductServices/IDL.aspx
} 
Table 1

Photometry of 1RXS J1730+03

\begin{tabular}{|c|c|c|c|c|c|}
\hline Date (UT) & HJD & Filter Name ${ }^{a}$ & Exposure Time (s) & Magnitude & Error $^{b}$ \\
\hline 20090417 & 54938.832278 & $g^{\prime}$ & 120 & 20.8 & 0.13 \\
\hline 20090417 & 54938.835488 & $i^{\prime}$ & 90 & 19.39 & 0.05 \\
\hline 20090417 & 54938.839953 & $i^{\prime}$ & 90 & 19.17 & 0.05 \\
\hline 20090417 & 54938.841212 & $g^{\prime}$ & 90 & 20.67 & 0.13 \\
\hline 20090417 & 54938.842469 & $i^{\prime}$ & 90 & 19.1 & 0.05 \\
\hline$\ldots$ & $\ldots$ & $\ldots$ & $\ldots$ & $\ldots$ & $\ldots$ \\
\hline 20090826 & 55069.691401 & $l f c i^{\prime}$ & 60 & 20.4 & 0.05 \\
\hline 20090826 & 55069.693218 & lf $c i^{\prime}$ & 60 & 20.04 & 0.06 \\
\hline
\end{tabular}

Notes.

${ }^{\text {a }}$ Filters $g^{\prime}, r^{\prime}$, and $i^{\prime}$ denote data acquired at P60 in the respective filters, $l f c i^{\prime}$ denotes data acquired in the $i^{\prime}$ band with the LFC at the Palomar 200" Hale telescope.

${ }^{\mathrm{b}}$ Relative photometry error. Values do not include an absolute photometry uncertainty of 0.16 mag in the $g^{\prime}$ band, 0.14 mag in the $r^{\prime}$ band, and $0.06 \mathrm{mag}$ in the $i^{\prime}$ band. Absolute photometry is derived from default P60 zero-point calibrations.

(This table is available in its entirety in a machine-readable form in the online journal. A portion is shown here for guidance regarding its form and content.)

Table 2

Photometry of Reference Stars for 1RXS J1730+03

\begin{tabular}{|c|c|c|c|c|c|}
\hline Identifier $^{a}$ & Right Ascension & Declination & $g^{\prime}$ Magnitude & $r^{\prime}$ Magnitude & $i^{\prime}$ Magnitude \\
\hline A & $17: 30: 01.47$ & $03: 38: 37.5$ & $15.861 \pm 0.003$ & $15.600 \pm 0.003$ & $15.341 \pm 0.003$ \\
\hline B & $17: 30: 00.97$ & $03: 37: 11.0$ & $17.372 \pm 0.009$ & $17.153 \pm 0.006$ & $16.872 \pm 0.008$ \\
\hline C, 10 & 17:30:11.04 & $03: 37: 17.0$ & $16.879 \pm 0.006$ & $16.691 \pm 0.005$ & $16.423 \pm 0.006$ \\
\hline $\mathrm{D}, 9$ & 17:30:08.92 & 03:38:03.0 & $18.161 \pm 0.016$ & $17.929 \pm 0.010$ & $17.660 \pm 0.013$ \\
\hline $\mathrm{E}$ & $17: 30: 04.02$ & $03: 38: 31.3$ & $18.518 \pm 0.020$ & $17.462 \pm 0.007$ & $16.811 \pm 0.007$ \\
\hline $\mathrm{F}$ & 17:30:03.67 & $03: 37: 26.4$ & $18.530 \pm 0.022$ & $18.158 \pm 0.012$ & $17.816 \pm 0.016$ \\
\hline $\mathrm{G}, 8$ & $17: 30: 07.87$ & 03:38:03.9 & $18.621 \pm 0.022$ & $18.259 \pm 0.012$ & $17.905 \pm 0.016$ \\
\hline $\mathrm{H}$ & $17: 30: 02.75$ & $03: 38: 32.8$ & $16.770 \pm 0.006$ & $16.081 \pm 0.004$ & $15.688 \pm 0.004$ \\
\hline I & $17: 29: 59.02$ & $03: 38: 43.7$ & $17.661 \pm 0.011$ & $17.302 \pm 0.007$ & $16.969 \pm 0.008$ \\
\hline 1 & $17: 30: 08.13$ & $03: 40: 28.3$ & $\ldots$ & $\ldots$ & $17.059 \pm 0.035$ \\
\hline 2 & 17:30:07.00 & $03: 39: 48.6$ & $\ldots$ & $\ldots$ & $19.855 \pm 0.043$ \\
\hline 3 & 17:30:10.11 & $03: 39: 32.4$ & $\ldots$ & $\ldots$ & $19.148 \pm 0.039$ \\
\hline 4 & $17: 30: 10.26$ & $03: 38: 59.4$ & $\ldots$ & $\ldots$ & $17.622 \pm 0.033$ \\
\hline 5 & $17: 30: 08.74$ & $03: 38: 39.0$ & $\ldots$ & $\ldots$ & $20.777 \pm 0.049$ \\
\hline 6 & $17: 30: 06.40$ & $03: 38: 29.5$ & $\ldots$ & $\ldots$ & $19.843 \pm 0.026$ \\
\hline 7 & $17: 30: 04.48$ & $03: 37: 57.9$ & $\ldots$ & $\ldots$ & $19.057 \pm 0.026$ \\
\hline 11 & $17: 30: 07.67$ & $03: 36: 45.4$ & $\ldots$ & $\ldots$ & $17.471 \pm 0.030$ \\
\hline 12 & 17:30:06.69 & $03: 36: 34.5$ & $\ldots$ & $\ldots$ & $16.339 \pm 0.044$ \\
\hline 13 & $17: 30: 13.30$ & $03: 36: 21.2$ & $\ldots$ & $\ldots$ & $18.006 \pm 0.030$ \\
\hline 14 & $17: 30: 07.03$ & $03: 36: 12.4$ & $\ldots$ & $\ldots$ & $18.085 \pm 0.048$ \\
\hline 15 & $17: 30: 09.24$ & $03: 35: 48.1$ & $\ldots$ & $\ldots$ & $17.392 \pm 0.055$ \\
\hline
\end{tabular}

Notes.

${ }^{a}$ The letters A-I denote stars used in photometry of P60 data and numbers 1-15 denote reference stars used in photometry of LFC images (Figure 1 and Section 2.1).

${ }^{\mathrm{b}}$ Relative photometry error. Values do not include an absolute photometry uncertainty of $0.16 \mathrm{mag}$ in the $g^{\prime}$ band, 0.14 mag in the $r^{\prime}$ band, and 0.064 mag in the $i^{\prime}$ band. Absolute photometry is derived using default P60 zero-point calibrations.

(This table is also available in a machine-readable form in the online journal.)

do not correct for this contamination. The statistical uncertainty in magnitudes is $\sim 0.2 \mathrm{mag}$ for P60 and $\sim 0.05 \mathrm{mag}$ for LFC, and the systematic uncertainty is 0.16 mag in the $g^{\prime}$ band, 0.14 mag in the $r^{\prime}$ band, and $0.06 \mathrm{mag}$ in the $i^{\prime}$ band.

The resultant light curves are shown in Figures 2-4. Table 1 provides the photometry.

\subsection{Spectroscopy}

We obtained optical and near-infrared spectra of 1RXS $\mathrm{J} 1730+03$ at various stages after outburst (Figure 5). The first optical spectra were taken 13 days after the first photometric epoch. We used the Low Resolution Imaging Spectrograph on the $10 \mathrm{~m}$ Keck-I telescope (LRIS; Oke et al. 1995), with upgraded blue camera (McCarthy et al. 1998; Steidel et al. 2004), covering a wavelength range from $3200 \AA$ to $9200 \AA$. We acquired more optical data 34 days after outburst, with the Double Beam Spectrograph on the $5 \mathrm{~m}$ Hale telescope at Palomar (DBSP; Oke \& Gunn 1982). We took five exposures spanning one complete photometric period, covering the 3500-10000 A wavelength range. We took late-time spectra covering just over one photometric period for the quiescent source with the upgraded LRIS. ${ }^{6}$ At this epoch, we aligned the slit at a position angle of $45^{\circ}$ to cover both the target and the contaminator, $3^{\prime \prime} .4$ to

\footnotetext{
6 http://www2.keck.hawaii.edu/inst/lris/lris-red-upgrade-notes.html
} 


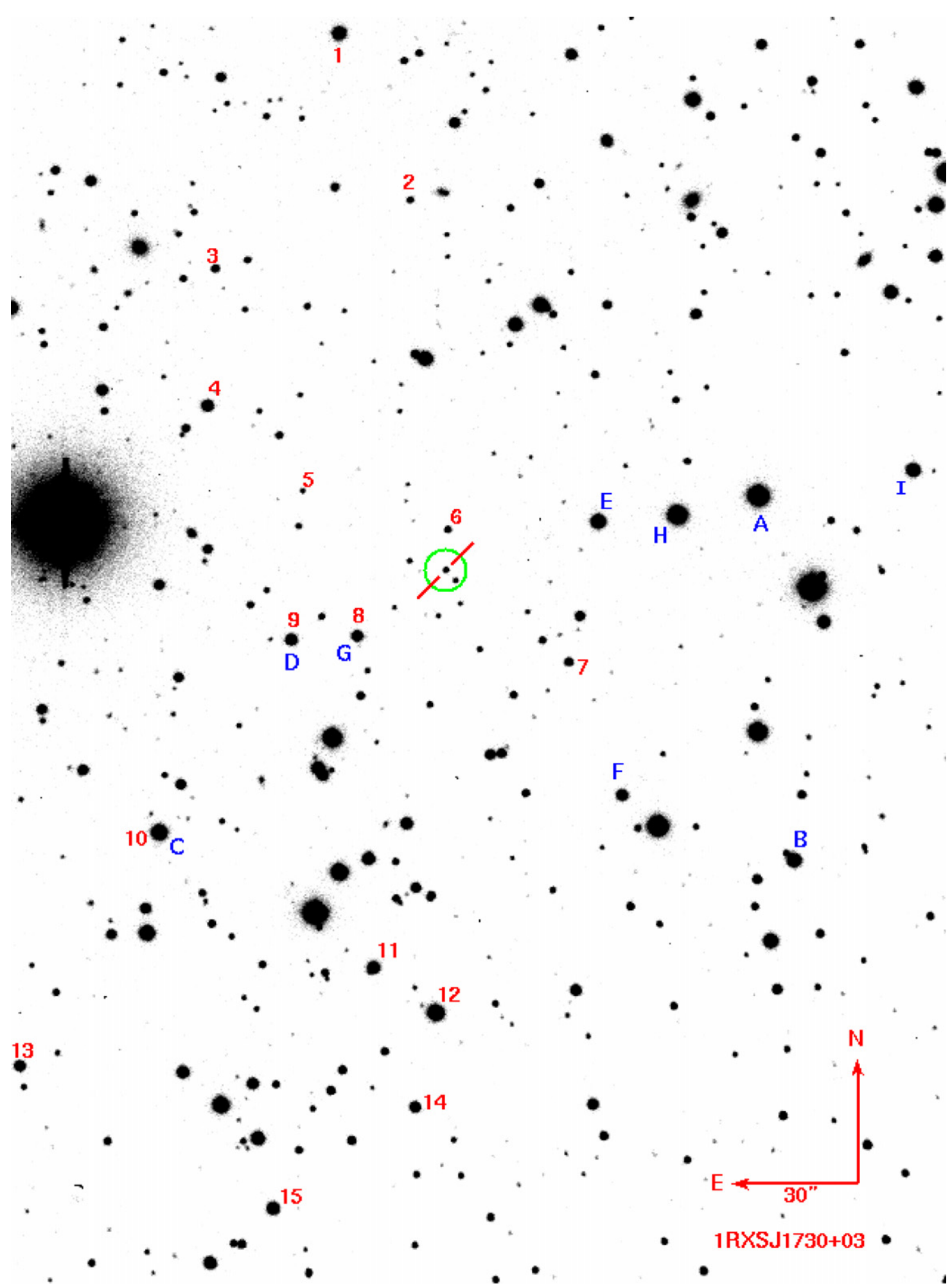

Figure 1. Finder chart for 1RXS J1730+03 $\left(\alpha=17^{\mathrm{h}} 30^{\mathrm{m}} 06^{\mathrm{s}} 19, \delta=+03^{\circ} 38^{\prime} 18^{\prime \prime} 8\right)$. This $i^{\prime}$ band image was acquired with the LFC at the $5 \mathrm{~m}$ Hale telescope at Palomar. Stars numbered 1-15 in red are used for relative photometry in LFC data. Stars labeled A-I in blue are used for relative photometry in P60 data (Section 2.1). The green circle shows the 5" extraction region used for calculating UVOT fluxes. The circle includes a contaminator, 3 ". 4 to the southwest of the target.

(A color version of this figure is available in the online journal.)

its southwest (Figure 1). We also obtained low-resolution $J$-band spectra with the Near Infrared Spectrograph on the $10 \mathrm{~m}$ Keck-II telescope (NIRSPEC; McLean et al. 1998). Twelve spectra of 5 minutes each were acquired, covering the wavelength region from $11500 \AA$ to $13700 \AA$. For details of the observing set up, see the notes to Table 5 later.
We analyzed the spectra using IRAF and MIDAS ${ }^{7}$ and flux calibrated them using appropriate standards. Wavelength solutions were obtained using arc lamps and with offsets determined from

7 Munich Image Data Analysis System,
http://www.eso.org/sci/data-processing/software/esomidas/ 
Table 3

Observation Log for Swift ToO Observations of 1RXS J1730+03

\begin{tabular}{|c|c|c|c|c|c|c|c|c|}
\hline Obs ID & $\begin{array}{l}\text { Start Date and Time } \\
\text { (UT) }\end{array}$ & Stop Time & $\begin{array}{c}\text { Exposure } \\
(\mathrm{s})\end{array}$ & Filter & $\begin{array}{l}\text { Wavelength } \\
(\AA)\end{array}$ & Magnitude & Flux & $\begin{array}{l}\text { Flux } \\
(\mu \mathrm{Jy})\end{array}$ \\
\hline 00035571001 & 2006 Feb 9 16:56:43 & $18: 42: 00$ & 1106 & $\begin{array}{l}\text { UVM2 } \\
\text { XRT }\end{array}$ & 2231 & $17.9 \pm 0.1$ & $\begin{array}{c}31 \pm 2^{\mathrm{b}} \\
0.02^{\mathrm{c}}\end{array}$ & $\begin{array}{c}51 \pm 3 \\
0.06(5 \mathrm{keV})\end{array}$ \\
\hline 00031408001 & 2009 May 3 18:28:56 & 19:02:12 & 1964 & UVM2 & 2231 & $20.44 \pm 0.21$ & $3.04 \pm 0.59^{\mathrm{b}}$ & $5.05 \pm 0.99$ \\
\hline 00031408002 & 2009 May 4 18:34:04 & $22: 12: 54$ & 4896 & UVW1 & 2634 & $20.14 \pm 0.10$ & $3.47 \pm 0.32^{b}$ & $8.04 \pm 0.75$ \\
\hline 00031408003 & 2009 May 6 03:02:21 & $15: 36: 33$ & 5621 & $\begin{array}{l}\text { UVW2 } \\
\text { XRT }\end{array}$ & 2030 & $21.48 \pm 0.17$ & $\begin{array}{l}1.38 \pm 0.22^{\mathrm{b}} \\
<0.002^{\mathrm{c}}\end{array}$ & $\begin{array}{c}1.93 \pm 0.30 \\
<0.006(5 \mathrm{keV})\end{array}$ \\
\hline
\end{tabular}

Notes.

${ }^{a}$ Effective wavelength for each filter for a Vega-like spectrum (Poole et al. 2008).

${ }^{\mathrm{b}}$ Flux in the units of $10^{-17} \mathrm{erg} \mathrm{cm}^{-2} \mathrm{~s}^{-1} \mathrm{~A}^{-1}$.

${ }^{c}$ Counts s ${ }^{-1}$ in $0.5-10 \mathrm{keV}$.
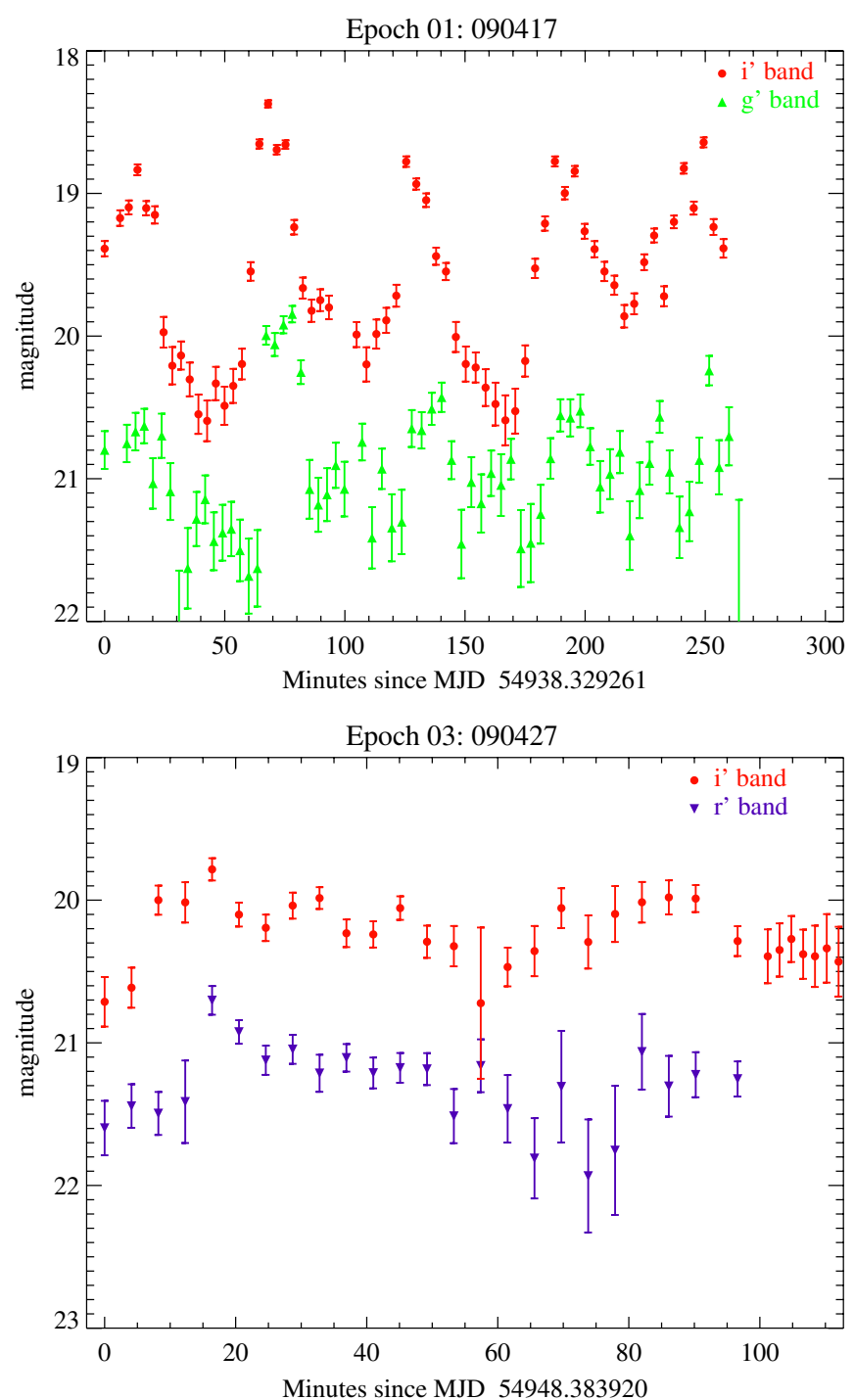

Figure 2. P60 photometry of 1RXS J1730+03. Top panel: Epoch 1 photometry in $g^{\prime}$ and $i^{\prime}$ bands. Bottom panel: Epoch 3 photometry in $r^{\prime}$ and $i^{\prime}$ bands. (A color version of this figure is available in the online journal.)

sky emission lines. Figure 6 shows an optical spectrum from each epoch, while the IR spectrum is shown in Figure 7.

The second LRIS epoch had variable sky conditions. Here, we extracted spectra of the aforementioned contaminator. This object is also a M dwarf, hence both target and contaminator spectra will be similarly affected by the atmosphere. We estimate the $i^{\prime}$ magnitude of the contaminator in each spectrum and compare it to the value measured from the LFC images to estimate and correct for the extinction by clouds.

\section{3. $X$-ray and UV Observations}

We observed the 1RXS J1730+03 with the X-ray telescope (XRT) and the UV-Optical Telescope (UVOT) on board the Swift X-ray satellite (Gehrels et al. 2004) during UT 2009 May 3-6 for a total of about $12.5 \mathrm{ks}$. The level-two event data were processed using Swift data analysis threads for the XRT (Photon counting mode; PC) using the HEASARC FTOOLS ${ }^{8}$ software package. The source was not detected in the X-ray band.

We follow the procedures outlined by Poole et al. (2008) for analyzing the UVOT data. The measured fluxes are given in Table 3. The contaminator is within the recommended $5^{\prime \prime}$ extraction radius. Hence, flux measurements are upper limits.

Shevchuk et al. (2009) had observed 1RXS J1730+03 on UT 2006 February 9 with the Swift satellite as a part of investigations of unidentified ROSAT sources. They detected the source with a count rate of 0.02 counts s ${ }^{-1}$. The best-fit power law has a photon index $\Gamma=1.8 \pm 0.5$ and a $0.5-10 \mathrm{keV}$ flux of $1.2 \times 10^{-12} \mathrm{erg} \mathrm{cm}^{-2} \mathrm{~s}^{-1}$. After converting to the ROSAT bandpass assuming the XRT model parameters, this value is approximately a factor of two lower than the archival ROSAT flux. They report a much higher UV flux in their observations, which suggests that the source was in an active state during their observations.

The column density inferred from the XRT data is low, $N_{\mathrm{H}} \sim 7 \times 10^{20} \mathrm{~cm}^{-2}$. From ROSAT data, this column density corresponds to $A_{V}=0.39$, which gives $A_{\mathrm{UVM} 2}=0.84(\mathrm{Cox}$ 2000). For comparison, Schlegel et al. (1998) give the Galactic dust extinction toward this direction $(l=26.7, b=19.7)$ to be $E(B-V)=0.141 \mathrm{mag}\left(A_{V}=0.44\right)$, corresponding to a column density of about $8 \times 10^{20} \mathrm{~cm}^{-2}$.

\section{NATURE OF THE COMPONENTS}

The optical spectra (Figure 6) show rising flux toward the red and blue ends of the spectrum: indicative of a hot (blue) and cool (red) component. The red part of the spectrum shows clear molecular features, characteristic of late-type stars. The blue component is devoid of any prominent absorption/emission features. From the overall spectral shape, we infer that 1RXS $\mathrm{J} 1730+03$ is a CV.

\footnotetext{
8 http://heasarc.gsfc.nasa.gov/ftools/, Blackburn (1995).
} 


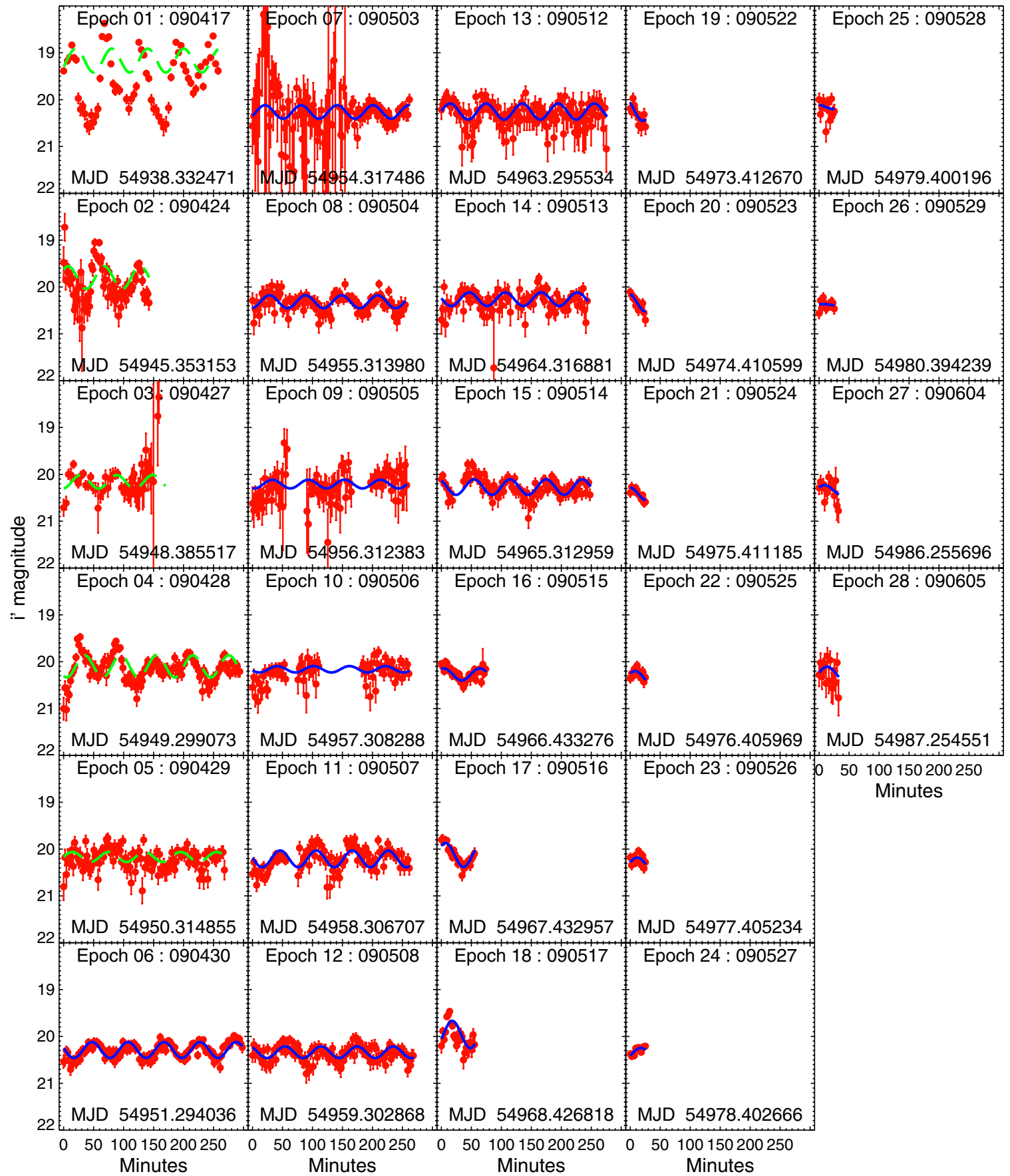

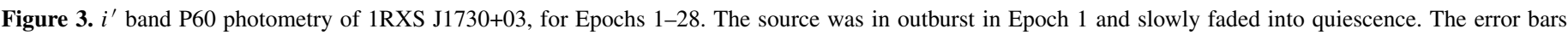

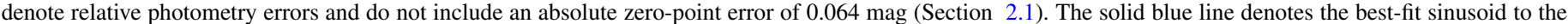
photometric data. The dashed green line is added to show the relative phase of the source in outburst, these epochs were not used for fitting the light curve.

(A color version of this figure is available in the online journal.)

\subsection{Red Component}

The red component of $1 \mathrm{RXS} \mathrm{J1730+03} \mathrm{is} \mathrm{typical} \mathrm{of} \mathrm{a} \mathrm{late-}$ type star. In Figure 8, we compare the red side spectrum of 1RXS J1730+03 with several M dwarfs. From the shape of the TiO bands at 7053-7861 $\AA$, we infer that the spectral type to be $\mathrm{M} 3 \pm 1$. This is consistent with the relatively featureless $J$-band spectrum (McLean et al. 2003). The spectral type indicates an effective temperature of $3400 \mathrm{~K}$ (Cox 2000). The presence of a sodium doublet at 8183/8195 ̊̊ implies a luminosity class V.
We also fit the spectrum with model atmospheres calculated by Munari et al. (2005). For late-type stars, these models are calculated in steps of $\Delta T=500 \mathrm{~K}, \Delta \log g=0.5$, and $\Delta[\mathrm{M} / \mathrm{H}]=0.5$. We use model atmospheres with no rotational velocity $\left(V_{\text {rot }}=0 \mathrm{~km} \mathrm{~s}^{-1}\right)$ and convolve them with a kernel modeled on the seeing, slit size, and pixel size. We ignore the regions contaminated by the telluric $A$ and $B$ bands $(7615 \AA$ and $6875 \AA$ ). The unknown contribution from the WD was fit as a low-order polynomial. We correct for extinction using $A_{V}=$ 0.39 from X-ray data (Section 2.3). To measure $\log g$, we use 


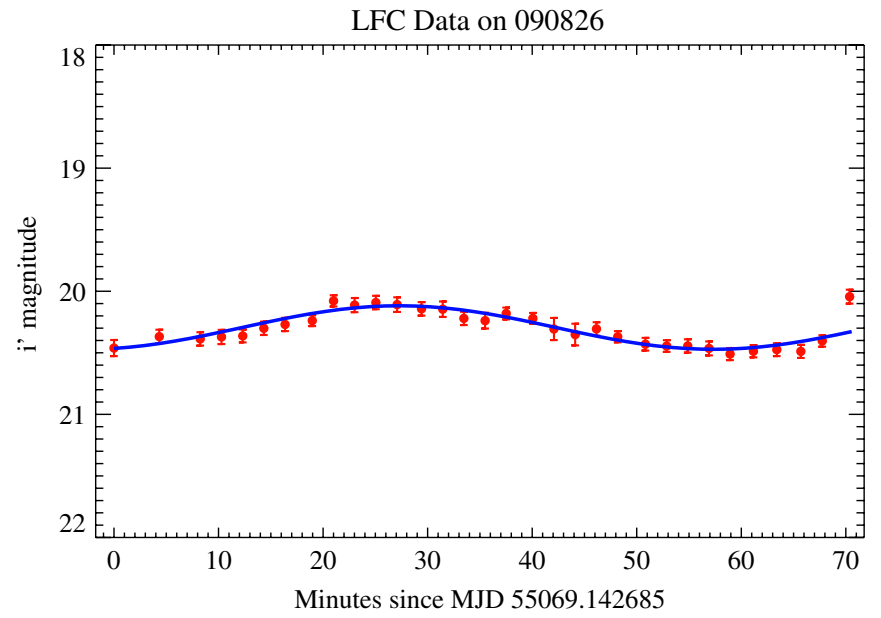

Figure 4. $i^{\prime}$ band LFC photometry of 1RXS J1730+03 in quiescence, 131 days after we first saw high variability. The variations have the same phase and period as observed during outburst. See Figure 3 for details.

(A color version of this figure is available in the online journal.)

the spectrum in the $8000-8700 \AA$ region, which is expected to have fairly little contamination from the blue component. This region includes the $\mathrm{Ca}$ II lines at $8498,8542 \AA$, and the $\mathrm{NaI}$ doublet, which are sensitive to $\log g$. We then fit the spectra in the 6700-8700 $\AA$ range to determine the temperature and metallicity. The best-fit model has $T=3500 \mathrm{~K}, \log g=5.0$, and solar metallicity, consistent with our determination of the spectral type.

Kolb et al. (2001) state that unevolved donors in CVs follow the spectral type-mass relation of the zero-age main sequence (ZAMS), as the effects of thermal disequilibrium on the secondary spectral type are negligible. For an M3 star, this yields a mass of $0.38 M_{\odot}$. As the secondaries evolve, the spectral type is no longer a good indicator of the mass and gives only an upper limit on the mass. The lower limit can simply be assumed to be the hydrogen-burning limit of $0.08 M_{\odot}$.
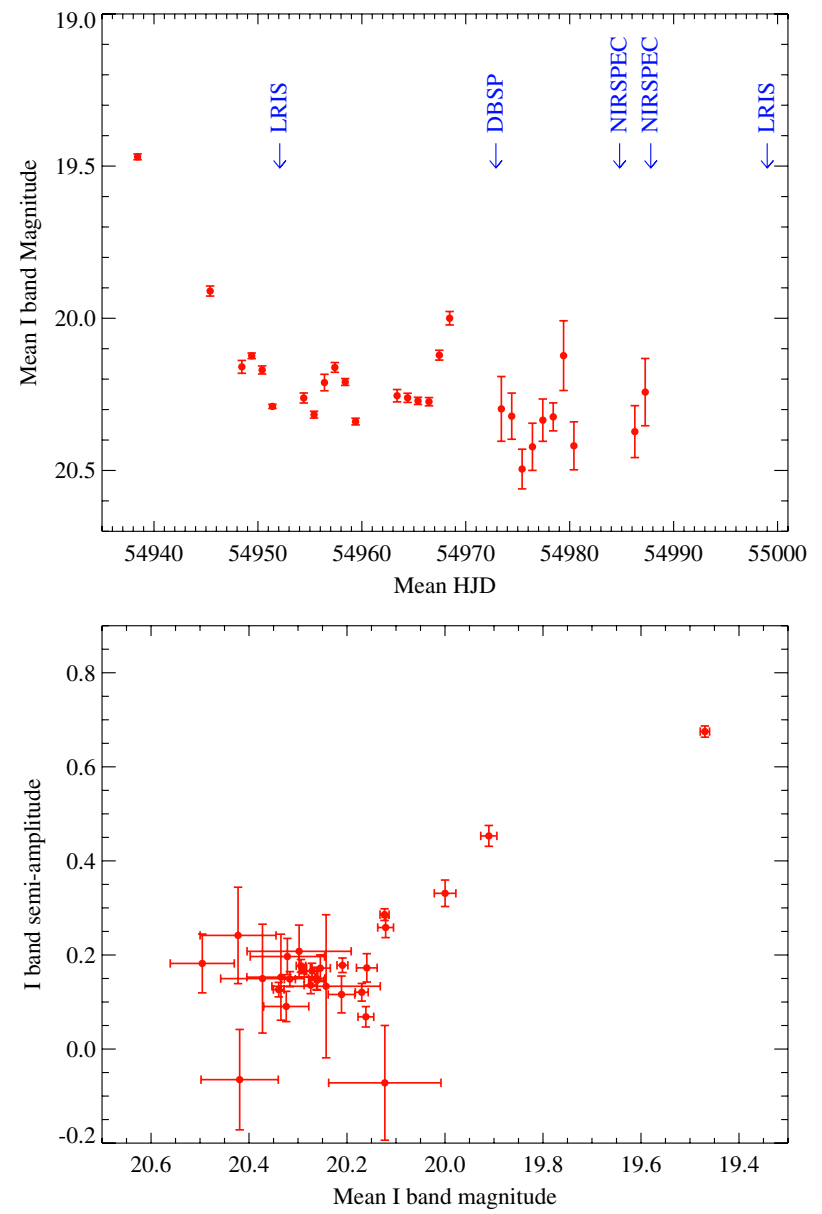

Figure 5. Photometric evolution of 1RXS J1730+03 in the $i^{\prime}$ band. Data for each epoch were fit with a sinusoid with the same period for all epochs. In contrast to Figures 3 and 4 , the phase was allowed to vary independently for each epoch. Top: average $i^{\prime}$ magnitude for each P60 observation epoch, as a function of time. Blue arrows mark spectroscopy epochs. Bottom: semi-amplitude of sinusoidal variations as a function of the corresponding mean $i^{\prime}$ magnitudes for each epoch. (A color version of this figure is available in the online journal.)

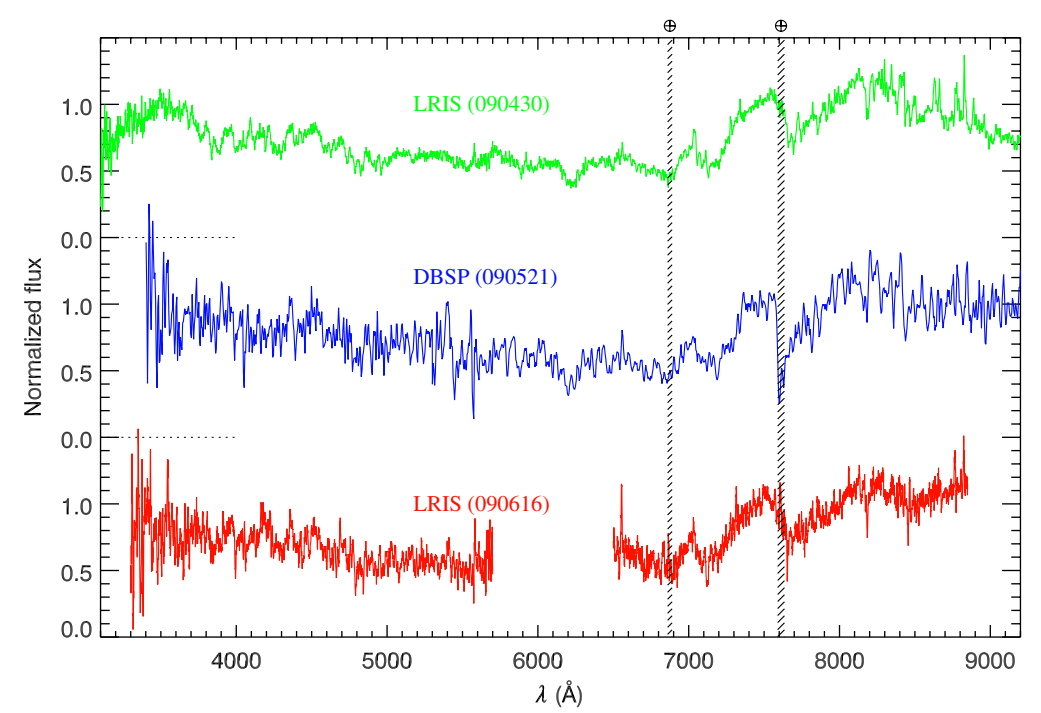

Figure 6. Temporal evolution of 1RXS J1730+03 spectra. Some spectra are not corrected for Telluric absorption, the affected regions are diagonally hatched. The first spectrum was obtained 13 days after we measured high variability, the second after 34 days, and the lowermost spectrum after 60 days. The spectra show broad Balmer features which evolve with time. The $\mathrm{M}$ dwarf features (TiO, Na I) are clearly seen at all times.

(A color version of this figure is available in the online journal.) 


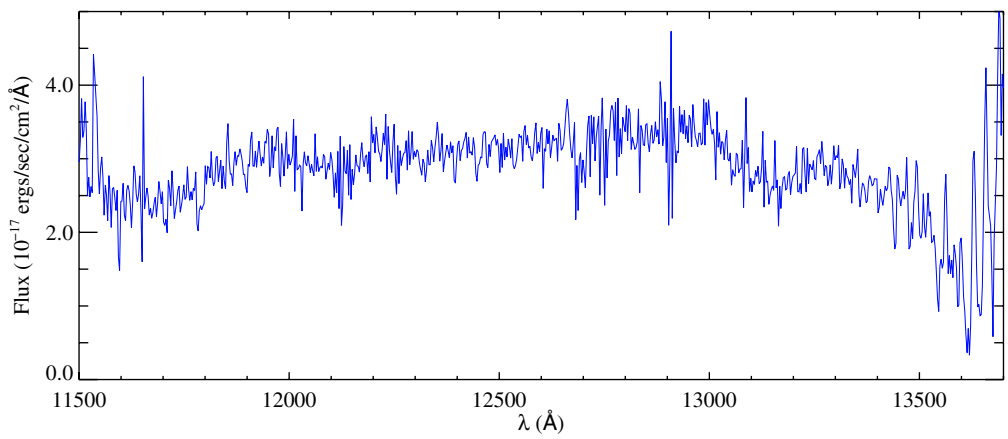

Figure 7. Keck NIRSPEC spectrum of 1 RXS J1730+03. The spectrum is flat and nearly featureless, as expected for early M stars.

(A color version of this figure is available in the online journal.)

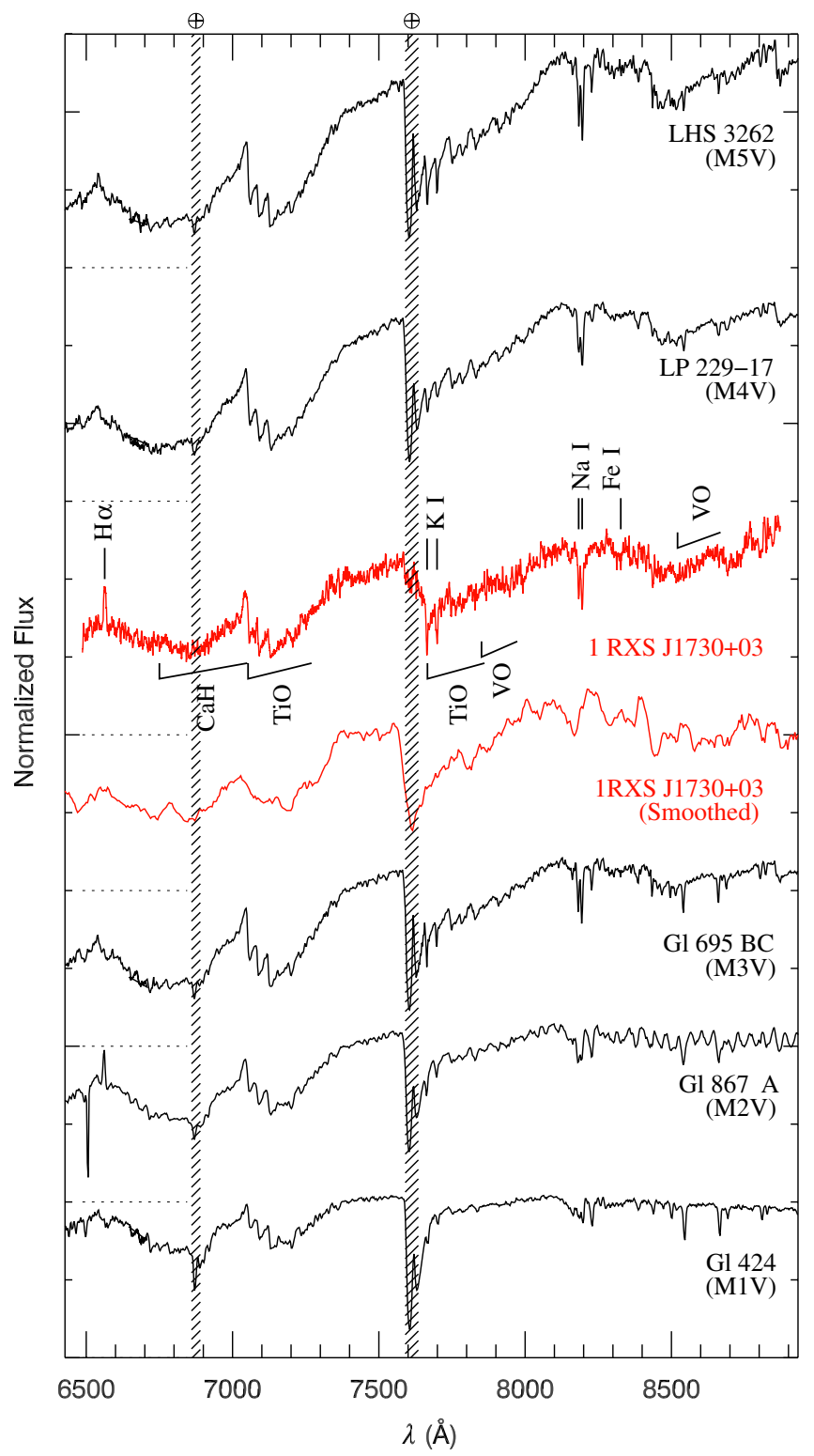

Figure 8. Comparison of red part of 1RXS J1730+03 spectrum with M dwarf spectra. Some spectra are not corrected for Telluric absorption, the affected regions are diagonally hatched. Prominent bands $(\mathrm{TiO}, \mathrm{CaH})$ and lines $(\mathrm{H} \alpha$, $\mathrm{Na}$ I) are marked. Comparing the shape of the TiO bands at 7053-7861 $\AA$ and the shape of the continuum redward of $8200 \AA$, we infer that spectral type of the red component to be M3. The presence of a sodium doublet at $8183 / 8195 \AA$ implies a luminosity class V.

(A color version of this figure is available in the online journal.)
Table 4

Locations of Cyclotron Harmonics

\begin{tabular}{cccc}
\hline $\begin{array}{c}\text { Harmonic } \\
\text { Number }\end{array}$ & $\begin{array}{c}\text { Measured } \\
\text { Wavelength } \\
(\AA)\end{array}$ & $\begin{array}{c}\text { Measured } \\
\text { Frequency } \\
(\mathrm{Hz})\end{array}$ & $\begin{array}{c}\text { Inferred } \\
\text { Wavelength } \\
(\AA)\end{array}$ \\
\hline 7 & 3540 & $8.4 \times 10^{14}$ & 3664 \\
6 & 4440 & $6.7 \times 10^{14}$ & 4275 \\
5 & 5180 & $5.7 \times 10^{14}$ & 5130 \\
4 & 6770 & $4.4 \times 10^{14}$ & 6413 \\
3 & 8225 & $3.6 \times 10^{14}$ & 8551 \\
2 & $\ldots$ & $\ldots$ & 12826 \\
1 & $\ldots$ & $\ldots$ & 25653 \\
\hline
\end{tabular}

Note. ${ }^{\text {a }}$ Calculated from the best-fit magnetic field strength, $B=42 \mathrm{MG}$.

\subsection{Blue Component}

The blue component of 1RXS J1730+03 is consistent with a highly magnetic WD. The blue spectrum is suggestive of a hot object, but does not show any prominent absorption/emission features. $\mathrm{H} \alpha$ is seen in emission, but other Balmer features are not detected. The spectrum (Figure 6) shows cyclotron humps, suggesting the presence of a strong magnetic field. The polar nature of the object is supported by the absence of an accretion disk and the transition from an active state to an off state in X-rays (Section 1, Section 2.3).

For analyzing the WD spectrum, we subtracted a scaled spectrum of the M dwarf GL 694 from the composite spectrum of 1 RXS J1730+03. The resultant spectrum (Figure 9) clearly shows cyclotron harmonics. The hump seen in the $J$-band spectrum (Figure 7) is also inferred to be a cyclotron harmonic. A detailed modeling of the magnetic field is beyond the scope of this work, but we use a simple model to estimate the magnetic field. We fit the cyclotron humps with Gaussians and measure the central wavelengths (Table 4). We then fit these as a series of harmonics and infer that the cyclotron frequency is $v_{\text {cyc }}=1.17 \times 10^{14} \mathrm{~Hz}$. The magnetic field in the emission region is given by $B=\left(v_{\text {cyc }} / 2.8 \times 10^{14} \mathrm{~Hz}\right) \cdot 10^{8} \mathrm{G}=42 \times 10^{6} \mathrm{G}$.

As a conservative error estimate, we consider the worst case scenario where our identified locations for the cyclotron humps are off by half the spacing between consecutive cyclotron harmonics. Using this, we estimate the errors on the magnetic field: $B=42_{-5}^{+6} \mathrm{MG}$.

\section{SYSTEM PARAMETERS}

\subsection{Orbit}

We use the best-fit (Munari et al. 2005) model atmosphere to measure radial velocities of the $\mathrm{M}$ dwarf. We vary the radial 


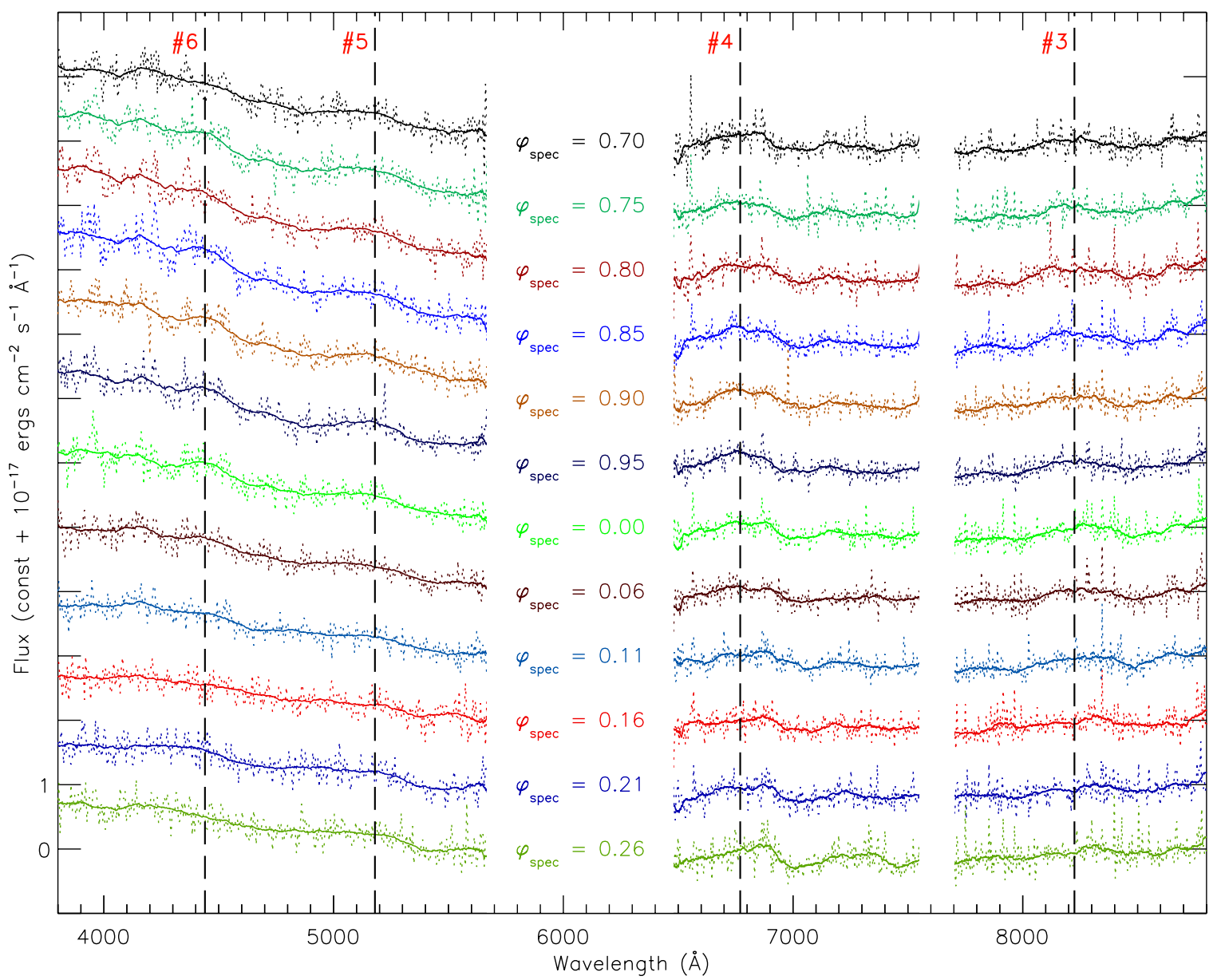

Figure 9. Blue component spectra of 1RXS J1730+03, showing the 12 late-time LRIS exposures (Section 2.2 and Table 5). The spectroscopic phase at mid-exposure is indicated for each spectrum. Dotted lines show that spectra are obtained by subtracting a scaled spectrum of GL 694 from spectra of this binary. The overlaid solid lines are smoothed versions of the same spectra. Vertical dashed cyclotron humps at $4440 \AA$, $5180 \AA, 6770 \AA$, and $8225 \AA$. The cyclotron harmonic numbers (3-6) are indicated in bold red. Two more cyclotron humps are seen in other spectra: a feature at $3540 \AA$ (Figure 6 ) and a $J$-band feature (Figure 7).

(A color version of this figure is available in the online journal.)

velocity of the model and minimize the $\chi^{2}$ over the $6500-8700 \AA$ spectral region, excluding the telluric $\mathrm{O}_{2}$ bands. After a first iteration, the spectra are re-fit to account for motion of the $\mathrm{M}$ dwarf during the integration time. For the 12 spectra taken at the second LRIS epoch, we also measure the radial velocity for the contaminator star on the slit and find it to be constant. This serves as a useful test for our radial velocity measurement procedure. The barycentric corrected velocities are given in Table 5 .

We fit a circular orbit $\left(v_{2}=\gamma_{2}+K_{2} \sin \left(\left[2 \pi\left(t-t_{0}\right)\right] / P\right)\right)$ to the measured velocities. We define the superior conjunction ${ }^{9}$ of the WD as phase 0 .

The 2009 June 16 spectroscopic data (Table 5) give an orbital period $P_{\text {est }}=123 \pm 3 \mathrm{~min}$. We then use the photometric variability (Section 4.2 ) to determine an accurate period in this range. Next, we refine the solution with velocity measurements from the other two spectroscopic epochs. The best-fit solution gives a period $P=120.2090 \pm 0.0013$ minutes and $K_{2}=$ $390 \pm 4 \mathrm{~km} \mathrm{~s}^{-1}$ (Table 6 and Figure 10).

Figure 11 shows sections of the last epoch spectra around $\mathrm{H} \alpha$ and the Na I doublet at 8184/8195 ̊. The Na I doublet clearly matches the velocity of the M dwarf in the orbit, but the $\mathrm{H} \alpha$ emission seems to have a smaller velocity amplitude. A possible explanation for this is that the $\mathrm{H} \alpha$ emission comes from the M

9 When the WD is furthest from the observer along the line of sight. dwarf surface that is closest to the WD, which may be heated by emission from the WD or the accretion region.

\subsection{Photometric Variability}

The light curve of 1RXS J1730+03 shows clear periodicity (Figures 2-4), with two peaks per spectroscopic period. Most of the photometric data were acquired in the $i^{\prime}$ band, which contains contribution from both the $\mathrm{M}$ dwarf and a cyclotron harmonic from the emission region near the WD.

A Fourier transform of the data (Figure 12) shows a strong peak at 60 minutes. We interpret this as a harmonic of the orbital period. To determine the exact period, we analyze the data as follows. As the object has a short orbital period, we convert all times to Heliocentric Julian Date for analysis. We fit a sinusoid $\left(m_{0}+m_{A} \sin \left(\left[2 \pi\left(t-t_{0}\right)\right] / P\right)\right)$ to each epoch, allowing $m_{0}$ and $m_{A}$ to vary independently for each epoch, but we use the same reference time $t_{0}$ and period $P$ for the entire fit. The mean magnitude is correlated with the amplitude (Figure 5). Note that the amplitude measured for the sinusoidal approximation for each epoch is always less than the actual peak-to-peak variations of the source during that epoch, as expected. The source is in the active state in the first few epochs, and we exclude epochs 1-5 from the fit to avoid contamination from the accretion stream and/or the accretion shock. 
Table 5

Radial Velocity of the M dwarf

\begin{tabular}{|c|c|c|c|}
\hline Heliocentric JD & $\begin{array}{c}\text { Exposure Time } \\
\text { (s) }\end{array}$ & $\begin{array}{l}\text { Barycentric Radial Velocity } \\
\qquad\left(\mathrm{km} \mathrm{s}^{-1}\right)\end{array}$ & Instrument \\
\hline 2454952.08721 & 1020 & $-398 \pm 34$ & LRIS $^{\mathrm{a}}$ \\
\hline 2454952.10067 & 1020 & $-195 \pm 22$ & LRIS $^{\mathrm{a}}$ \\
\hline 2454952.11179 & 600 & $109 \pm 16$ & LRIS $^{\mathrm{a}}$ \\
\hline 2454972.92923 & 600 & $55 \pm 92$ & DBSP $^{b}$ \\
\hline 2454972.93818 & 900 & $-258 \pm 112$ & DBSP $^{b}$ \\
\hline 2454972.94896 & 900 & $-324 \pm 69$ & $\mathrm{DBSP}^{\mathrm{b}}$ \\
\hline 2454972.95956 & 900 & $-430 \pm 105$ & $\mathrm{DBSP}^{\mathrm{b}}$ \\
\hline 2454972.96875 & 600 & $-42 \pm 150$ & $\mathrm{DBSP}^{\mathrm{b}}$ \\
\hline 2454998.99566 & 210 & $-421 \pm 11$ & LRIS $^{\mathrm{c}}$ \\
\hline 2454998.99969 & 300 & $-438 \pm 9$ & LRIS $^{c}$ \\
\hline 2454999.00418 & 300 & $-417 \pm 9$ & LRIS $^{c}$ \\
\hline 2454999.00850 & 300 & $-361 \pm 9$ & LRIS $^{\mathrm{c}}$ \\
\hline 2454999.01283 & 300 & $-266 \pm 10$ & LRIS $^{\mathrm{c}}$ \\
\hline 2454999.01715 & 300 & $-163 \pm 12$ & LRIS $^{c}$ \\
\hline 2454999.02147 & 300 & $-32 \pm 12$ & LRIS $^{c}$ \\
\hline 2454999.02580 & 300 & $98 \pm 15$ & LRIS $^{c}$ \\
\hline 2454999.03012 & 300 & $186 \pm 10$ & LRIS $^{\mathrm{c}}$ \\
\hline 2454999.03448 & 300 & $290 \pm 17$ & LRIS $^{c}$ \\
\hline 2454999.03880 & 300 & $336 \pm 13$ & LRIS $^{c}$ \\
\hline 2454999.04317 & 300 & $344 \pm 13$ & LRIS $^{\mathrm{c}}$ \\
\hline
\end{tabular}

Notes.

a LRIS (Oke et al. 1995), with upgraded blue camera (McCarthy et al. 1998; Steidel et al. 2004). Settings—Blue side: $3200-5760 \AA ̊$, grism with 400 grooves $\mathrm{mm}^{-1}$, blaze $3400 \AA$, dispersion $1.09 \AA \mathrm{pixel}^{-1}$, and $R \sim 700$. Red side: $5450-9250 \AA$, grating with 400 grooves $\mathrm{mm}^{-1}$, blaze $8500 \AA$, dispersion $1.16 \AA$ pixel $^{-1}$, and $R \sim 1600$. Dichroic: $5600 \AA$, slit: $1^{\prime \prime} .0$.

b Double Spectrograph on the $5 \mathrm{~m}$ Hale telescope at Palomar (Oke \& Gunn 1982). Settings_-Blue side: $3270-5700 \AA$, grating with 600 lines $\mathrm{mm}^{-1}$, blaze $4000 \AA$, dispersion $1.08 \AA \mathrm{pixel}^{-1}$, and $R \sim 1300$. Red side: 5300-10200 $\AA$, grating with 158 grooves $\mathrm{mm}^{-1}$, blaze $7500 \AA$, dispersion $4.9 \AA$ pixel $^{-1}$, and $R \sim 600$. Dichroic: D55 (5500 Å), slit: 1.'5.

c LRIS, with upgraded blue and red cameras; http://www2.keck.hawaii.edu/ inst/lris/lris-red-upgrade-notes.html. Settings-Blue side: 3300-5700 A, grism with 400 grooves $\mathrm{mm}^{-1}$, blaze $3400 \AA$, dispersion $1.09 \AA \mathrm{pixel}^{-1}$, and $R \sim 700$. Red side: $6760-8800 \AA$, grating with 831 grooves $\mathrm{mm}^{-1}$, blaze $8200 \AA$, dispersion $0.58 \AA$ pixel $^{-1}$, and $R \sim 1800$. Dichroic: $5600 \AA$, slit: $1^{\prime \prime}$.0.

Table 6

Orbital Velocity Parameters of the M dwarf

\begin{tabular}{lr}
\hline \hline Parameter & \multicolumn{1}{c}{ Value } \\
\hline$\gamma_{2}\left(\mathrm{~km} \mathrm{~s}^{-1}\right)$ & $-48 \pm 5$ \\
$K_{2}\left(\mathrm{~km} \mathrm{~s}^{-1}\right)$ & $390 \pm 4$ \\
$t_{0}(\mathrm{HJD})$ & $54998.9375 \pm 0.0003$ \\
$P(\min )$ & $120.2090 \pm 0.0013$ \\
\hline
\end{tabular}

The best-fit solution is overplotted in blue in Figures 3 and 4 . Since epochs $1-5$ were not included in the fit, a sinusoid is overplotted in dashed green to indicate the expected phase of the variations. The best-fit period is $60.1059 \pm 0.0005$ minutes. This formally differs from the spectroscopically determined orbital period by $2.1 \sigma$. However, this error estimate includes only statistical errors. There is some, difficult to determine, systematic error component in addition, so we do not claim any significant inconsistency.

Periodic photometric variability for 1 RXS J1730+03 can be explained as a combination of two effects: cyclotron emission from the accretion region and ellipsoidal modulation. The active state is characterized by a higher mass transfer rate from the donor to the WD and results in higher cyclotron emission. This emission is beamed nearly perpendicular to
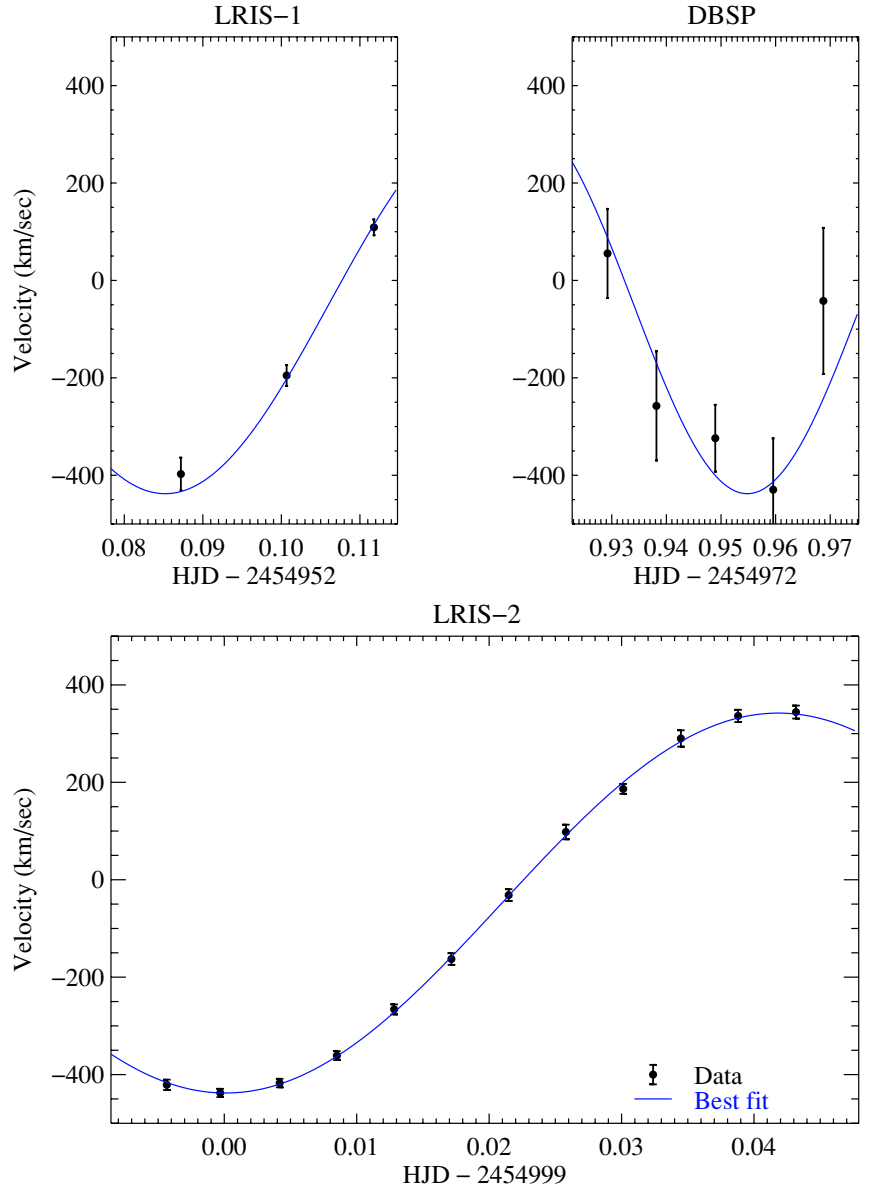

Figure 10. Velocity measurements 1 RXS J1730+03. The best-fit solution gives $P=120.2090 \pm 0.0013$ minutes, $\gamma_{2}=-48 \pm 5 \mathrm{~km} \mathrm{~s}^{-1}$, and $K_{2}=$ $390 \pm 4 \mathrm{~km} \mathrm{~s}^{-1}$ (Section 4.1).

(A color version of this figure is available in the online journal.)

the magnetic field lines, creating an emission fan beam. For high inclination systems, the observer crosses this fan beam twice, causing two high amplitude peaks per orbit (Figure 2). In the active state when the emission is dominated by cyclotron radiation, the minimum leads the superior conjunction by $\sim 47^{\circ}$.

In ellipsoidal modulation, the photometric minima coincide with the superior conjunction. It is observed that in quiescence, the photometric minimum leads the superior conjunction of the WD by $\sim 14^{\circ}$. This suggests that the $0.29 \pm 0.13$ mag variation is caused by a combination of ellipsoidal modulation and cyclotron emission from the accretion region.

\subsection{Mass Ratio}

The semi-amplitude of the M dwarf radial velocity, K2, gives a lower limit on the mass of the WD. For a circular orbit, one can derive from Kepler's laws that

$$
M_{1, \min }=\frac{P K_{2}^{3}}{2 \pi G}=0.52 M_{\odot} .
$$

Tighter constraints can be placed on the individual component masses $M_{1}, M_{2}$ by considering the geometry of the system. Eggleton (1983) expresses the volume radius $R_{2}$ of the 


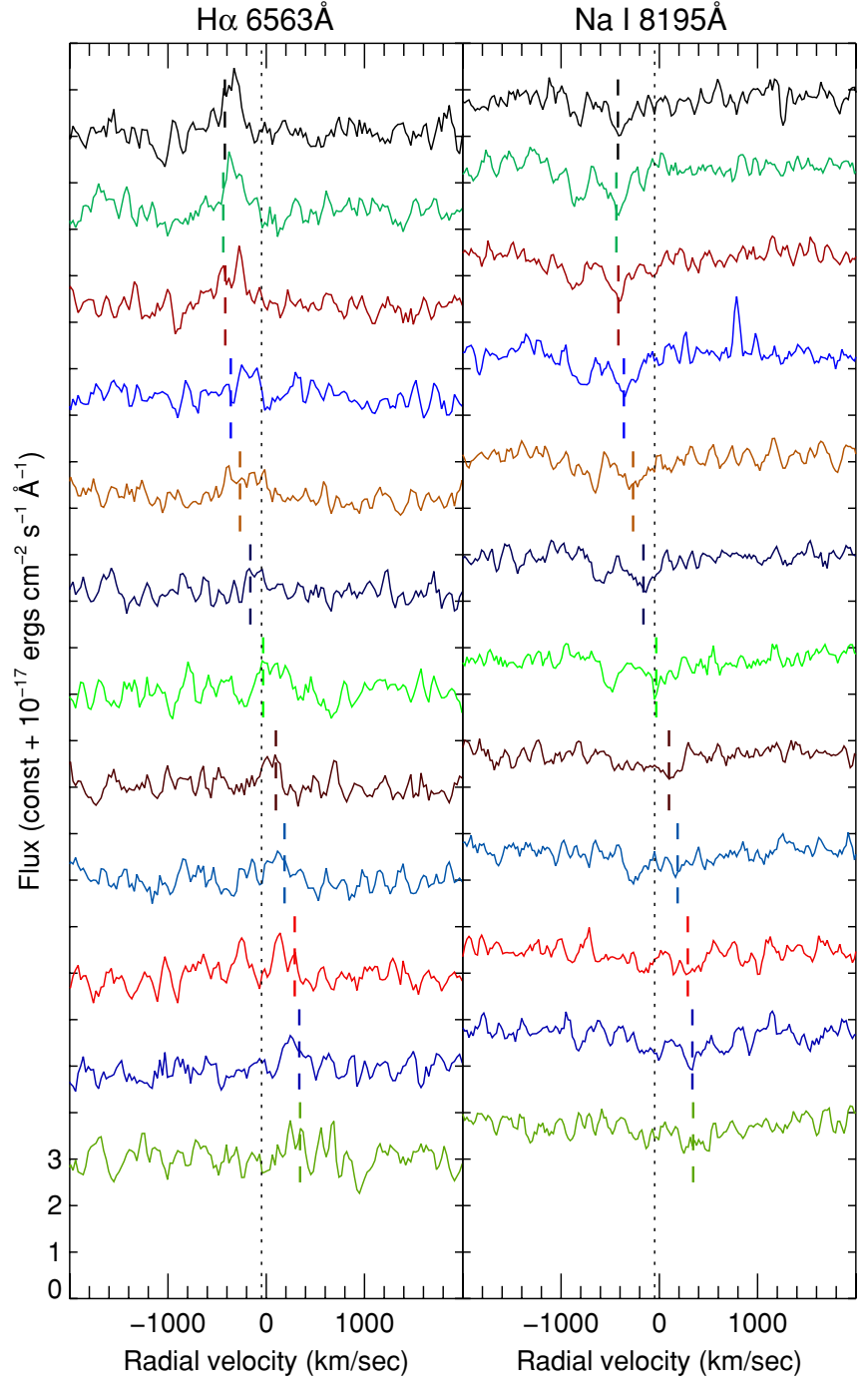

Figure 11. Velocity modulation of $\mathrm{H} \alpha$ and the $8184 / 8195 \AA$ Na I doublet The spectra are offset by $2 \times 10^{-17} \mathrm{erg} \mathrm{cm}^{-2} \mathrm{~s}^{-1}$ for clarity. The wavelengths are converted into velocities using the rest wavelengths $6563 \AA$ and $8195 \AA$, respectively. The vertical dotted line marks the radial velocity of the binary barycenter. The short dashed lines mark the radial velocities measured by fitting the complete spectrum. The Na I absorption follows the radial velocity of the M dwarf, but $\mathrm{H} \alpha$ seems to have a smaller velocity amplitude.

(A color version of this figure is available in the online journal.)

secondary in terms of the mass ratio $q=M_{2} / M_{1}$ :

$$
\frac{R_{2}}{a}=\frac{0.49 q^{2 / 3}}{0.6 q^{2 / 3}+\ln \left(1+q^{1 / 3}\right)},
$$

where the separation of the components is given by $a=$ $\left[P^{2} G\left(M_{1}+M_{2}\right) / 4 \pi^{2}\right]^{1 / 3}$. Thus for a fixed period $P, R_{2}$ depends only on $M_{2}$. The radius of the WD is much smaller than that of the $\mathrm{M}$ dwarf. Hence, the $\mathrm{M}$ dwarf will eclipse the accretion region if the inclination of the system is $i<\sin ^{-1}\left(R_{2} / a\right)$. Figures 3 and 4 show that we do not detect any eclipses in the system.

Figure 13 shows the allowed region for 1RXS J1730+03 in a WD mass-M dwarf mass phase space. The orange dotted region is excluded as the orbital velocity would be greater than the measured projected velocity. The red hatched region is excluded by non-detection of eclipses. The allowed mass of the primary ranges from the minimum mass $\left(M_{1}>0.52 M_{\odot}\right)$ to the Chandrasekhar limit. The mass of the secondary is bounded above by the ZAMS mass for an M3 dwarf $\left(M_{2}=0.38 M_{\odot}\right)$.

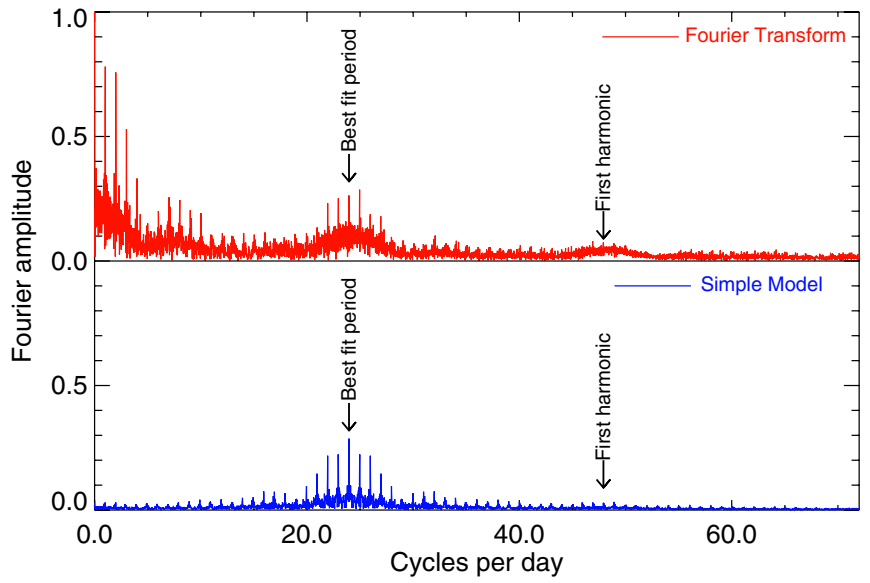

Figure 12. Upper panel: Fourier transform of all $i^{\prime}$ data. Lower panel: expected Fourier transform for a pure sinusoidal variations with a period of 60.11 minutes, obtained by scaling and convolving the Fourier transform of the window function with a delta function corresponding to the best-fit period.

(A color version of this figure is available in the online journal.)

For a given $M_{2}$ and a known orbital period, we can determine the radius of the secondary using Equation (2) and can calculate the surface gravity $(\log g)$. For $0.38 M_{\odot} \gtrsim M_{2} \gtrsim 0.05 M_{\odot}$, $\log g$ ranges from 5.1 to 4.8. This is consistent with $\log g=5.0$ for the best-fit M dwarf spectrum (Section 3.1).

The donor stars in CVs are expected to corotate. For 1RXS J1730+03, the highest possible rotational velocity $v \sin i$ is $\sim 160 \mathrm{~km} \mathrm{~s}^{-1}$ for a $0.38 M_{\odot}, 0.27 R_{\odot} \mathrm{M}$ dwarf, and a $1.1 M_{\odot}$ WD (Figure 13). $v \sin i$ will be lower if the WD is heavier or if the $\mathrm{M}$ dwarf is lighter. To measure rotational broadening in the spectra, we use a higher resolution $(R=20,000)$ template of the best-fit model from Zwitter et al. (2004). We take a template with zero rotation velocity and broaden it to different rotational velocities using the prescription by Gray (2005). Then we use our fitting procedure (Section 3.1) to find the best-fit value for $v \sin i$. For this measurement, we use only the 12 relatively highresolution spectra from the second LRIS epoch (UT 2009 June 16). The weighted $v \sin i$ from the 12 spectra is $97 \pm 22 \mathrm{~km} \mathrm{~s}^{-1}$, but the measurements show high scatter, with a standard deviation of $54 \mathrm{~km} \mathrm{~s}^{-1}$. We compared our broadened spectra with rotationally broadened spectra computed by Zwitter et al. (2004) and found that our methods systematically underestimate $v \sin i$ by $\sim 20 \mathrm{~km} \mathrm{~s}^{-1}$. We do not understand the reason for this discrepancy, hence do not feel confident enough to use this value in our analysis. A reliable measurement of $v \sin i$ will help to better constrain the masses of the two components.

\subsection{Distance}

We estimate the distance to 1RXS $\mathrm{J} 1730+03$ as follows. Our fitting procedure (Section 3.1) corrects for extinction and separates the WD and M dwarf components of the spectra. We correct for varying sky conditions by using a reference star on the slit. The ratio of measured flux to the flux of the best-fit model atmosphere $(T=3500 \mathrm{~K}, \log g=5.0)$ is

$$
\frac{f_{\text {measured }}}{f_{\text {model }}}=\left(\frac{R_{2}}{d}\right)^{2}=(6.1 \pm 1.6) \times 10^{-23},
$$

where $d$ is the distance to the source and $R_{2}$ is given by Equation (2).

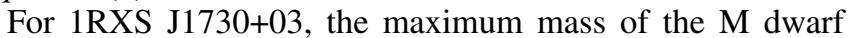
is $0.38 M_{\odot}$, and the corresponding radius is $R_{2}=0.27 R_{\odot}$. 


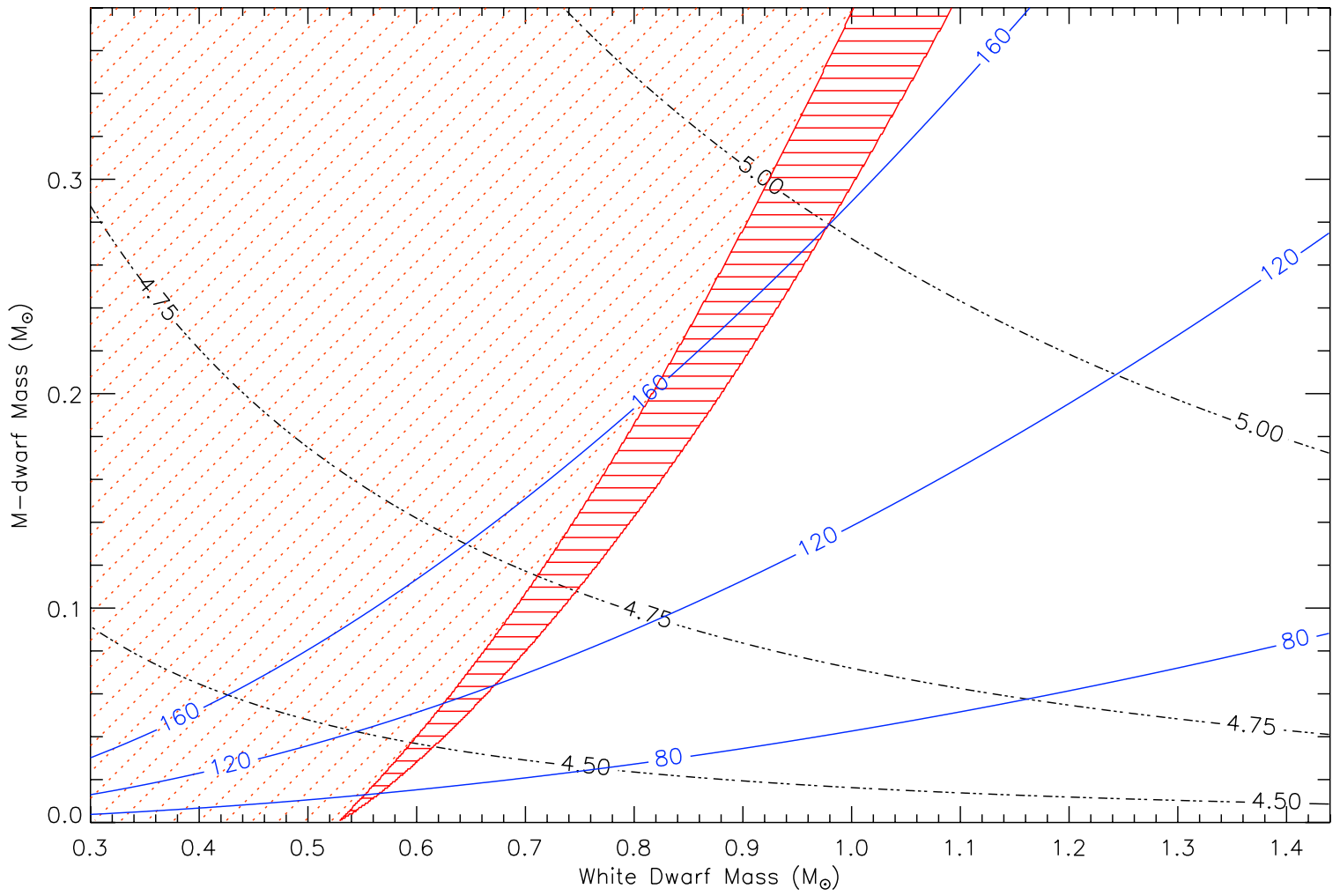

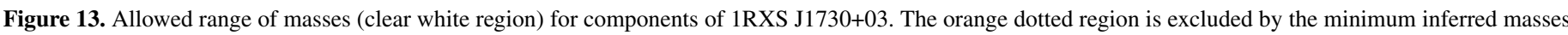

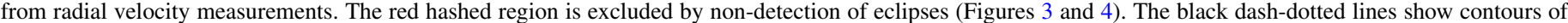

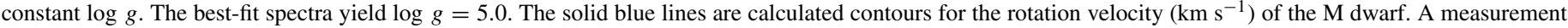
of $v \sin i$ will help to constrain masses of the components.

(A color version of this figure is available in the online journal.)

Equation (3) then gives $d=800 \pm 110$ pc. This calculation assumes the largest possible $\mathrm{M}$ dwarf radius, hence is an upper limit to distance. If the $\mathrm{M}$ dwarf is lighter, say $0.1 M_{\odot}$, we get $R_{2}=0.17 R_{\odot}$, yielding $d=500 \pm 70$ pc.

\section{CONCLUSION}

1RXS J173006.4+033813 is a polar CV, similar to known well-studied systems like BL Hyi, ST LMi, and WW Hor in terms of the orbital period, magnetic field, and variability between active and quiescent states. This source is notable for the highly symmetric nature and high amplitude of the doublepeaked variation in the active state. This suggests a relatively high angle between the rotation and magnetic axes. Polarimetric observations of the source would help to better constrain the magnetic field geometry of the system.

Most polars are discovered due to their highly variable $\mathrm{X}$-ray flux. However, we mounted a follow-up campaign for 1RXS J1730+03 due to its unusual optical variability properties. This suggests that current and future optical synoptic surveys, such as $\mathrm{PTF}^{10}$ (Law et al. 2009) and LSST can uncover a large sample of polars by cross-correlating optically variable objects with the ROSAT catalog.

We sincerely thank the anonymous referee for detailed comments on the paper. We thank N. Gehrels for approving the Target of Opportunity observation with Swift and the Swift team for executing the observation. We also thank V. Anupama,

\footnotetext{
$10 \mathrm{http}: / / \mathrm{www}$. astro.caltech.edu/ptf
}

L. Bildsten, T. Marsh, G. Nelemans, E. Ofek, and P. Szkody for useful discussions while writing the paper.

This research has benefitted from the M, L, and T dwarf compendium housed at DwarfArchives.org and maintained by Chris Gelino, Davy Kirkpatrick, and Adam Burgasser.

Some of the data presented herein were obtained at the W.M. Keck Observatory, which is operated as a scientific partnership among the California Institute of Technology, the University of California, and the National Aeronautics and Space Administration. The Observatory was made possible by the generous financial support of the W.M. Keck Foundation.

Facilities: PO:1.5m, Hale (LFC, DBSP), Keck:I (LRIS), Keck:II (NIRSPEC), Swift

\section{REFERENCES}

Blackburn, J. K. 1995, in ASP Conf. Ser. 77, Astronomical Data Analysis Software and Systems IV, ed. R. A. Shaw, H. E. Payne, \& J. J. E. Hayes (San Francisco, CA: ASP), 367

Cenko, S. B., et al. 2006, PASP, 118, 1396

Cox, A. N. 2000, Allen's Astrophysical Quantities (4th ed.; Berlin: Springer)

Cropper, M. 1990, Space Sci. Rev., 54, 195

Denisenko, D. V., Kryachko, T. V., \& Satovskiy, B. L. 2009, ATel, 2014, 1

Eggleton, P. P. 1983, ApJ, 268, 368

Gehrels, N., et al. 2004, ApJ, 611, 1005

Gray, D. F. 2005, in The Observation and Analysis of Stellar Photospheres, ed. D. F. Gray (3rd ed.; Cambridge: Cambridge Univ. Press) (http://www.cambridge.org/us/catalogue/catalogue.asp?isbn=0521851866)

Hellier, C. 2001, Springer-Praxis Books in Astronomy \& Space Sciences (Chichester: Springer-Praxis)

Hellier, C. 2002, in ASP Conf. Ser. 261, The Physics of Cataclysmic Variables and Related Objects, ed. B. T. Gänsicke, K. Beuermann, \& K. Reinsch (San Francisco, CA: ASP), 92 
Kolb, U., King, A. R., \& Baraffe, I. 2001, MNRAS, 321, 544

Landsman, W. B. 1993, in ASP Conf. Ser. 52, Astronomical Data Analysis Software and Systems II, ed. R. J. Hanisch, R. J. V. Brissenden, \& J. Barnes (San Francisco, CA: ASP), 246

Law, N. M., et al. 2009, PASP, 121, 1395

McCarthy, J. K., et al. 1998, Proc. SPIE, 3355, 81

McLean, I. S., McGovern, M. R., Burgasser, A. J., Kirkpatrick, J. D., Prato, L., \& Kim, S. S. 2003, ApJ, 596, 561

McLean, I. S., et al. 1998, Proc. SPIE, 3354, 566

Mighell, K. J. 1999, in ASP Conf. Ser. 172, Astronomical Data Analysis Software and Systems VIII, ed. D. M. Mehringer, R. L. Plante, \& D. A. Roberts (San Francisco, CA: ASP), 317

Munari, U., Sordo, R., Castelli, F., \& Zwitter, T. 2005, A\&A, 442, 1127

Oke, J. B., \& Gunn, J. E. 1982, PASP, 94, 586
Oke, J. B., et al. 1995, PASP, 107, 375

Poole, T. S., et al. 2008, MNRAS, 383, 627

Ramsay, G., Cropper, M., Wu, K., Mason, K. O., Córdova, F. A., \& Priedhorsky, W. 2004, MNRAS, 350, 1373

Schlegel, D. J., Finkbeiner, D. P., \& Davis, M. 1998, ApJ, 500, 525

Shevchuk, A. S., Fox, D. B., Turner, M., \& Rutledge, R. E. 2009, ATel, 2015, 1

Simcoe, R. A., Metzger, M. R., Small, T. A., \& Araya, G. 2000, BAAS, 32, 758

Steidel, C. C., Shapley, A. E., Pettini, M., Adelberger, K. L., Erb, D. K., Reddy, N. A., \& Hunt, M. P. 2004, ApJ, 604, 534

Voges, W., et al. 1999, A\&A, 349, 389

Warner, B. 1995, Cambridge Astrophysics Series (Cambridge: Cambridge Univ. Press)

Zwitter, T., Castelli, F., \& Munari, U. 2004, A\&A, 417, 1055 